\title{
Application of the penalty coupling method for the analysis of blood vessels
}

\author{
Ian Owens Pericevic - Moji Moatamedi \\ Stress Analysis Research Group, Institute for Materials Research \\ University of Salford Manchester M5 4W UKT \\ m.moatamedi@salford.ac.uk
}

\begin{abstract}
Due to the significant health and economic impact of blood vessel diseases on modern society, its analysis is becoming of increasing importance for the medical sciences. The complexity of the vascular system, its dynamics and material characteristics all make it an ideal candidate for analysis through fluid structure interaction (FSI) simulations. FSI is a relatively new approach in numerical analysis and enables the multi-physical analysis of problems, yielding a higher accuracy of results than could be possible when using a single physics code to analyse the same category of problems. This paper introduces the concepts behind the Arbitrary Lagrangian Eulerian (ALE) formulation using the penalty coupling method. It moves on to present a validation case and compares it to available simulation results from the literature using a different FSI method. Results were found to correspond well to the comparison case as well as basic theory.

RÉSUMÉ. En raison de leur impact sanitaire et économique dans les sociétés modernes, l'analyse des maladies cardio-vasculaires revêt une importance croissante sur le plan médical. La complexité du système vasculaire, sa dynamique et les caractéristiques de ses matériaux constitutifs font qu'il se prête idéalement aux analyses par simulation d'interactions fluide-structure (IFS). L'IFS est une approche relativement récente permettant l'analyse numérique de systèmes en conditions multiphysiques, les résultats obtenus étant plus précis que ceux fournis par l'application d'un code monophysique au même système. Cet article présente les concepts associés à une formulation Arbitraire Lagrange-Euler (ALE) avec couplage par pénalisation. Un cas de validation est ensuite considéré. Un bon accord est trouvé entre les résultats obtenus par la formulation présentée dans cet article et ceux issus d'une formulation ISF existante.
\end{abstract}

KEYWORDS: blood vessel, penalty coupling, fluid structure interaction, multi-physics.

MOTS-CLÉS: vaisseau sanguin, couplage par pénalité, interaction fluide structure, multiphysiques.

DOI:10.3166/REMN.16.537-548@ 2007 Lavoisier, Paris. Tous droits réservés

REMN - 16/2007. Fluid structure interaction, pages 537 to 548 


\section{Introduction}

According to the World Health Organisation (WHO), in 2002 alone there were 16.7 million deaths globally related to various forms of cardiovascular diseases (CVD) (Mackay et al., 2004). This makes CVD the world's leading killer. Over $60 \%$ of these deaths occurred in the developing world. Many others who live with various forms of CVD suffer a large decrease in quality of life due to a reduction in mobility and loss of earnings. It is difficult to estimate the full economic implications resulting from CVD, however they are substantial. One study estimated that in 2003 alone, CVD cost the European Union $€ 169$ billion, with healthcare accounting for $62 \%$ of costs (Leal et al., 2006). A large proportion of these cardiovascular diseases find their origins in the blood vessels.

The onset and progression of most vascular diseases is influenced by a complex interaction of biochemical and biomechanical factors. The exact mechanisms of most of these diseases remain uncertain and are currently being extensively studied in research laboratories and universities across the world. In recent years, strong links between physical conditions in vessels and certain diseases have been established. The significant nonlinearities associated with the behaviour of blood vessels in the human body limit the practical application of analytical methods in their evaluation. Accurate measurement of physical parameters in vivo using experimental methods is extremely difficult if not impossible. Much progress has been made using medical imaging equipment, but challenges remain. These factors have opened the door to numerical simulations which are able to give predictions of the physical behaviour of blood vessels based on satisfactory input information. However, much work is necessary to increase efficiency and improve results.

A blood vessel is a highly nonlinear system where any changes in the fluid dynamics can bring on changes to the wall dynamics and vice versa. In many cases the approach of considering the flow and structural responses independently of each other soon becomes insufficient and some way of evaluating the full fluid structure interaction becomes a necessity. Analytical solutions of fluid structure interaction problems related to blood vessels have been around for many years now. The first person to propose a formula for the velocity of pressure waves in a thin elastic tube was (Young, 1808). Womersley (1957) investigated the dynamic response of a tube with a sinusoidal flow and defined an analytical solution for the flow field. Analytical solutions soon become impractical when realistic geometries and physiological conditions are considered, which creates the need for numerical simulation.

In the past, commercial codes were limited to either fluid or structural analyses and the modelling of fluid structure interaction was only available in specialised academic codes. Computational fluid dynamics has extensively been applied to examine flow conditions of blood through various parts of the cardiovascular system. These simulations were gradually expanded to include results for the wall shear stress distributions of the vessel (Gijsen et al., 1999). At the same time finite 
element simulations were applied to examine phenomena of the vessel wall. Design and optimization of medical devices and cardiovascular implants, such as stents, has also been studied using finite elements (Migliavacca et al., 2005). With the development of computational power and commercial software more accurate representations became available. It was accepted that the mechanics of the vascular system were quite different than other engineering problems (Salehi, 2004). The numerical analysis of blood vessels is a highly complex task and requires the use of multi-physics software capable of describing the full extent of the problem. Continuous development of fluid structure interaction methods is necessary to enable a more physiologically realistic representation of the system in question.

Over the years, several researchers have developed algorithms that coupled commercial codes for fluid and structure analysis. By 1998 Zhao (Zhao et al., 1999) had coupled the fluid dynamics code CFX with the structural dynamics code ABAQUS via their own iterative algorithm for their study of blood vessels. The rapid expansion in availability of low-cost computing has further led to the development of several methods to study fluid structure interaction through simulations which are implemented in a number of numerical codes. Most of these were created with purely engineering applications in mind, such as the study of aeroelastic effects in aircraft or the effect of underwater explosions on submarine hulls. Since then fluid structure interaction simulations have diversified and found applications in determining the correlations between disease and physical phenomena.

In this paper we explore the fluid structure interaction of blood vessels using the Arbitrary Lagrangian Eulerian formulation. The fluid structure interface between the fluid domain and the vascular wall is applied using the penalty coupling method through the commercial finite element code LS-DYNA (LSTC Inc, Livermore, CA, USA). The case study adopted for this research is the one presented by (Kuntz and Menter, 2004), who used a significantly different fluid structure interaction approach to solve the problem. The validation simulates the onset of a sinusoidal pressure wave is applied to the inlet of a linearly elastic tube of finite dimensions. The tube is constrained at both ends in the axial direction, causing an oscillation of the tube wall in the radial direction. The induced oscillation is then evaluated and compared with the results of Kuntz and Menter.

The basic concepts of ALE and penalty coupling when applied to numerical fluid structure interaction are explained in section two of this article. The method used by Kuntz and Menter is also considered in this section. Section 3 introduces the MoensKorteweg equation, the basic theory governing sinusoidal flow through an elastic tube, and a parameter used as a benchmark in this study. The validation study is presented in Section 4 of this paper, along with relevant results. 


\section{Applied numerical methods}

\subsection{The arbitrary Lagrangian Eulerian formulation}

Fluid structure interaction is a more recent development in numerical analysis and is the multi-physical combination of computational fluid mechanics (CFD) and computational structural mechanics (CSM). An FSI system can be defined as a complex interaction between the dynamic responses of an elastic structure and a viscous fluid. A fluid structure-interaction problem is, in fact, a three field problem. In addition to the solution of the computational fluid dynamics and computational structural mechanics domains, the computational mesh dynamics (CMD) must be considered (Alderson and Zamir, 2004). Three common formulations exist for discretizing fluid and structural problems. These are the Lagrangian, Eulerian and ALE formulations.

Structural finite element analysis typically uses the Lagrangian formulation. Here the mesh is fixed to, and defined by, the material it is describing. As the continuum moves and deforms in space, the mesh follows it and any individual material point remains exclusively within its original element. It is only possible to have one material in a single element. This type of mesh is suitable for models where deformations remain small or moderate. A major problem exists when it comes to describing a flowing fluid using a Lagrangian mesh. As a material point moves further away from its initial position, the mesh distortion becomes uncontrollable. Therefore, the use of this type of mesh is usually restricted to problems in structural mechanics and fluids with very low deformations.

The Eulerian formulation is mostly applied for CFD analyses. It uses a mesh which is fixed in space, through which material points move. In other words, the material flows through the mesh. More than one material can be present within a single element with this type of mesh. Eulerian meshes are used for the solution of fluid dynamics and high deformation structural problems. Solving structural mechanics problems may however become excessively computationally expensive as the mesh has to be very fine to capture the full material response at the boundaries. A further disadvantage compared to a Lagrangian formulation is that external material boundaries are not mapped by the mesh itself and must be calculated separately.

The concept of ALE was first developed in 1974 when (Hirt et al., 1974) presented a solution for the Navier-Stokes equations that was both Eularian and Lagrangian in formulation. As the name suggests, the Arbitrary Lagrangian Eulerian formulation is based on the principle that mesh motion can be chosen arbitrarily. In fact, the Lagrangian and Eulerian formulations are only two special cases of ALE. ALE can be considered as a set of algorithms that perform automatic remapping of the mesh. The ALE formulation consists of a Lagrangian time-step followed by an advection step. The advection step is when the mesh is mapped from a distorted Lagrangian mesh to an undistorted mesh. The great advantage of this method lies in 
the ability of the mesh to modify itself to follow material boundaries at large deformations without the need for re-meshing, as would be the case for high deformations when using a Lagrangian description. Thus, the ALE captures material responses while eliminating the need for a high mesh density as with an Eulerian formulation.

The main difference between an Eulerian and an ALE mesh is in the method the new undistorted mesh is defined. In an Eulerian formulation the solution is mapped back onto the original mesh in its undistorted state. This creates the effect of a fixed mesh through which the material points move. With ALE, the solution is mapped from the distorted mesh back onto a newly generated mesh. The nodes of this new mesh are placed at an arbitrary position between their locations on the original mesh and their locations on the distorted mesh.

The interdependency of ALE, Lagrange and Euler formulations can easily be demonstrated in mathematical terms by examining the Navier-Stokes equations. As mentioned, the reference coordinates in an ALE mesh move independently of the material motion. Assuming the velocity of the material is denoted by $u$, the velocity of reference coordinates by $v$ and the relative velocity between the material and the mesh by $w$, a relationship between the material and reference time derivatives can be written:

$$
\frac{\partial f^{r}}{\partial t}=\dot{f}-w_{i} \frac{\partial f}{\partial x_{i}}, \quad w_{i}=u_{i}-v_{i}
$$

where $f^{r}$ is the function $f$ expressed in terms of reference coordinates, $r$.

In any system mass, momentum and energy must be conserved. For a Lagrangian system this can be expressed mathematically through the Navier-Stokes equations as:

$$
\begin{aligned}
& \dot{\rho}=\rho-\frac{\partial u_{i}}{\partial x_{i}} \\
& \rho \dot{u}=\sigma_{i j, j}+\rho f_{i} \\
& \rho \dot{e}=\sigma_{i j} u_{i, j}+\rho f_{i} u_{i}
\end{aligned}
$$

The ALE equations of conservation of mass, momentum and energy are obtained by substituting the relationship between the material and reference time derivatives (Equation [1]) into Equations [2] thru [4]: 


$$
\begin{aligned}
& \frac{\partial \rho^{r}}{\partial t}=-\rho \frac{\partial u_{i}}{\partial x_{i}}-w_{i} \frac{\partial \rho}{\partial x_{i}} \\
& \rho \frac{\partial u^{r}}{\partial t}=\left(\sigma_{i j, j}+\rho f_{i}\right)-\rho w_{j} \frac{\partial u_{i}}{\partial x_{i}} \\
& \rho \frac{\partial e^{r}}{\partial t}=\left(\sigma_{i j, j} u_{i, j}+\rho f_{i} u_{i}\right)-\rho w_{j} \frac{\partial e_{i}}{\partial x_{i}}
\end{aligned}
$$

By setting the reference system velocity, $v$, to zero the relative velocity between the reference system and the material becomes the material velocity, $u$, yielding the standard Euler conservative equations.

\subsection{The penalty coupling method}

The simulation of certain problems may require that one part of the domain is described by a Lagrangian mesh, while the other is described by an Eulerian mesh or an ALE mesh. A typical fluid structure interaction problem would be solved using a contact formulation. In such cases the Lagrangian and Eulerian/ALE meshes are distinct and are bound by a contact interface algorithm. Contact algorithms calculate the forces applied from the fluid to the structure and vice versa at each step in time. A simplified ALE formulation is necessary to describe the physics of the fluid, insuring it remains in contact with the deforming Lagrangian mesh. This type of formulation is limited by the amount the mesh can be distorted and is generally used for simpler fluid structure interaction problems. Contact methods are further limited by the fact they preserve the momentum and not the energy in the system.

In many fluid structure interaction problems it is necessary for a Lagrangian mesh to move through an Eulerian or ALE mesh. Some form of interaction or "coupling" between the two meshes must then be defined. In LS-DYNA this coupling is achieved with the penalty method. The penalty coupling algorithm searches for the fluid structure interface at each time step and calculates the coupling forces on the nodes of the structure (slave nodes) and those of the fluid (master nodes). At the same time, flow through the Lagrangian mesh must be restricted by the algorithm to maintain the physics of the problem.

The penalty method concept is best understood by considering the sketch below (Figure 1): 
time $=\mathbf{t}_{\mathrm{n}}$

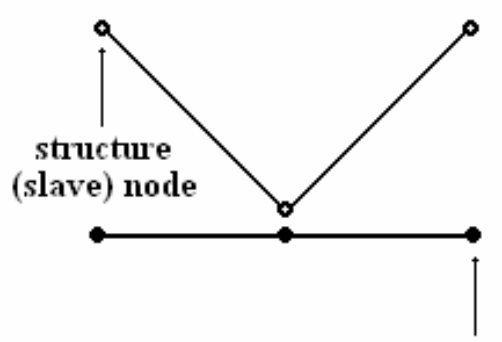

fluid (master)

node time $=\mathbf{t}_{n+1}$

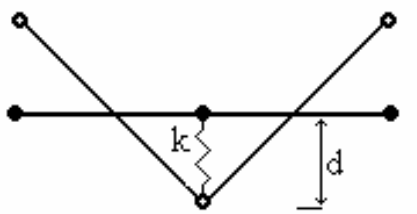

Figure 1. Penalty coupling for fluid structure interaction applications

When a structure is immersed into a fluid element, a resisting force is imposed on the slave node and is proportional to its penetration through the master element, thus returning the penetrating node to the interface between the two meshes. The force is characterised by springs placed between the master and the slave nodes:

$$
F=-k d
$$

where, $k$ is the stiffness and $d$ is the distance over which it acts.

The spring stiffness is given as:

$$
k=p_{f} \frac{K A}{V}
$$

where $K$ is the bulk modulus of the master material, $V$ is the volume of the master element, $A$ is the area of the master segment and $p_{f}$ is the scale factor for the interface stiffness.

To insure equilibrium is satisfied, the penalty force must be applied to the slave and master nodes in opposite directions. Energy is conserved with the penalty method; however, a slight disadvantage is the possible occurrence of stability problems. A detailed description of the penalty method is given by (Aquelet et al., 2005).

The greatest advantage of using the penalty coupling method in comparison to other fluid structure interaction techniques is its ability to accommodate for very large deformations without the risk of simulation failure, or a significant increase in solve time. 


\subsection{The Kuntz and Menter FSI approach}

In Kuntz and Menter, the fluid structure interaction is achieved by iteratively coupling two single-physics codes. The finite element structural code ANSYS 8.0 is coupled with the general-purpose finite volume CFD code CFX 5.7. Both codes are products by ANSYS Inc., Canonsburg, PA, USA. The actual coupling is achieved through the third-party code MpCCI release 2.0 (Fraunhofer Institute for Algorithms and Scientific Computing, Sankt Augustin, Germany).

The main concept behind the coupling is that internal data is exchanged between the two programs as they iterate through their respective solutions. Information is passed between the two through coupling ports controlling the data import and export. Interpolation of data on the fluid structure interface is carried out by the coupling library MpCCI. Once the structural and fluid solutions have both converged, the solution moves on in time to the next time step. This coupling method allows for either one-way or two-way coupling, depending on what type of problem is being analysed. Data can be transferred between the two codes at a specified number of time steps or during the internal coefficient loops. The coupled codes can run either simultaneously or alternating. The user is given full control over which options to use by manipulating how the ports are used.

\section{The analytical solution for the wave speed}

A blood vessel can be described as an elastic tube through which blood ejected by the heart flows. The periodical ejections of blood from the heart cause the propagation of a pressure wave (the pulse) down the vascular network at a much greater velocity than the blood itself. The wave propagates through the tube in the form of a radial displacement of the blood vessel wall. The speed at which the wave travels down a section of blood vessel is known as the wave speed. The wave speed is highly dependent on the elastic behavior of the vessel wall, which will limit its velocity. As the wave continues down the network of vessels, it becomes damped and distorted due to the wall mechanics, vessel geometry and reflected waves.

Assuming blood is an inviscid and incompressible fluid flowing through a thin vessel constrained in the direction of the flow; the Moens-Korteweg equation (Moens, 1878) gives the resulting wave speed $\left(\mathrm{c}_{\mathrm{o}}\right)$ :

$$
c_{0}=\sqrt{\frac{E h}{2 R \rho}}
$$

Where, $E$ is the Young's Modulus of a pipe of internal radius $R$, with a wall thickness $h$, carrying a fluid of density $\rho$. Errors caused as a result of assuming a thin tube in [10] can be estimated by using the Bergel correction (Bergel, 1961), which accounts for the thickness through Poisson's ratio (v). The difference between the two is given as: 


$$
\left(\frac{c^{\prime}}{c_{0}}\right)^{2}=\frac{(2-\gamma)}{\left[2-2 \gamma\left(1-v-2 v^{2}\right)+\gamma^{2}\left(1-v-2 v^{2}\right)-2 v^{2}\right.}
$$

where, $\gamma$ is the ratio of wall thickness and tube outer radius.

Simplifying this expression and incorporating it into Equation [10], the wave speed (c') becomes:

$$
c^{\prime}=\sqrt{\frac{E h}{2 R \rho\left(1-v^{2}\right)}}
$$

\section{Validation study}

The validation is based on a tube of $80 \mathrm{~mm}$ length with an internal diameter of $4 \mathrm{~mm}$, and a wall thickness of $0.12 \mathrm{~mm}$. The vessel wall is described as linearly elastic with a density of $1075 \mathrm{~kg} / \mathrm{m}^{3}$, a Poisson's ratio of 0.45 and an initial Young's modulus of $3 \mathrm{MPa}$. The fluid domain is defined as an incompressible Newtonian model of water, a realistic assumption for modelling blood. A density of $1000 \mathrm{~kg} / \mathrm{m}^{3}$ and a viscosity of $0.001 \mathrm{~Pa}$.s were therefore chosen. Translational constraints are applied on the vessel wall at the inlet on in the direction of flow and in all degrees of freedom at the exit. The onset of a sinusoidal pressure wave is simulated at the vessel entrance by a linearly increasing pressure from zero to $352 \mathrm{~Pa}$ over a time period of $8 \mathrm{~ms}$.

The problem was set-up in LS-DYNA with 68,480 elements describing the fluid domain, while the blood vessel wall was discretised using 4848 Belythchko-Tsay elements. The structure was immersed in the fluid mesh, leaving a zone of vacuum into which the vessel could expand (Figure 2). An interaction between the two domains was defined through the penalty coupling method using 3 quadrature points, which eliminated any leakage through the structure. This mesh was decided upon after a comprehensive mesh density study. In numerical terms, this case is equivalent to that used by Kuntz and Menter.

A comparison is made between numerical results and Moens-Korteweg theory by varying Young's modulus between the given values. Radial displacement results at different locations along the tube were extracted at $1 \mathrm{~ms}$ intervals from LS-DYNA and the wave speed was evaluated from these by a program written in MATLAB (MathWorks, Natick, MA, USA).

The range of Young's modulus used for this study is representative of small arteries assuming linear elasticity. Figure 3 shows numerical results obtained for the pulse wave speed as from the current model in LS-DYNA as a function of Young's modulus and compares them with theory, both Moens-Korteweg (Equation [12]) and 
numerical results from Kuntz and Menter. Young's modulus is varied between $0.1 \mathrm{MPa}$ and $3 \mathrm{MPa}$ in the study. Data for $0.1 \mathrm{MPa}$ is not available from Kuntz and Menter. As can be seen from Figure 3, the results from LS-DYNA compare very well with those predicted by theory. Furthermore, the results coincide with those obtained from the ANSYS-CFX coupling. Both codes tend to slightly over-predict the wave speed. The maximum differences between the results obtained by this study and those expected from theory are less than $10 \%$.

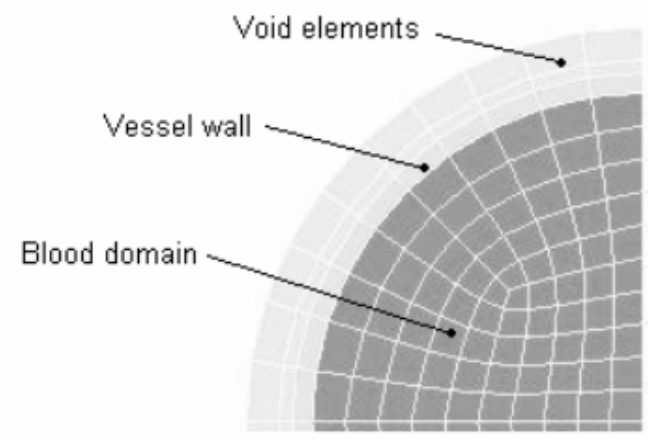

Figure 2. Definition of the blood vessel problem using the Arbitrary Lagrangian Eulerian formulation and penalty coupling

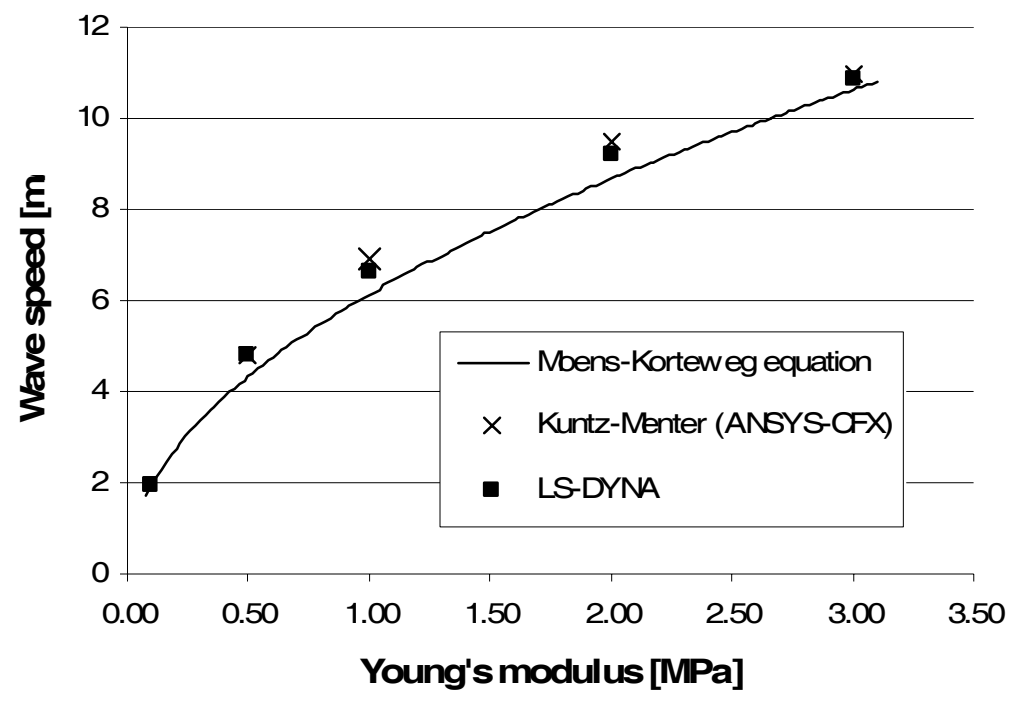

Figure 3. Wave speed as function of Young's modulus: comparison of numerical results and idealised theory 


\section{Conclusions}

This article has examined the use of the penalty coupling method in the simulation of blood vessels dynamics. The main concepts behind the Arbitrary Lagrangian Eulerian formulation and penalty coupling have been presented. A simple validation case looking at the wave speed in response to a pressure input within a blood vessel was set-up. This case was directly comparable to another numerical simulation found in the literature with a completely different approach to fluid structure interaction. It was found that the numerical approach applied here gave nearly identical results to the comparison simulations. Both fluid structure interaction methods were seen to slightly over predict theoretical results. In conclusion, it can be said that the method employed here was successful.

\section{Acknowledgements}

The authors wish to gratefully acknowledge the Engineering and Physics Research Council (EPSRC) for making this research possible.

\section{References}

Alderson H., Zamir M., "Effects of stent stiffness on local haemodynamics with particular reference to wave reflections", Journal of Biomechanics, vol. 37, n 3, 2004, p. 339-348.

Aquelet N., Souli M., Olovsson L., "Euler-Lagrange coupling with damping effects. Application to slamming problems. Computational", Methods in Applied Mechanical Engineering, 2005.

Bergel D.H., The visco-elastic properties of the arterial wall, PhD Thesis, University of London, 1961.

Gijsen F.J.H., Van de Vosse F.N., Janssen J.D., "The influenceof the non-Newtonian properties of blood on the flow of large arteries: steady flow in a carotid bifurcation model", Journal of Biomechanics, vol. 32, 1999, p. 601-608.

Hirt C.W., Amsden A.A., Cook J.L., "An Arbitrary Lagrangian Eulerian Computing model for all flow speeds", Journal of Computational Physics, vol. 14, 1974, p. 227-253.

Kuntz M., Menter F.R., "Simulation of fluid-structure interactions in aeronautical applications", European Congress on Computational Methods in Applied Sciences and Engineering (ECCOMAS 2004).

Leal J., Lengo-Fernandez R., Gray A., Petersen S., Rayner M., "Economic burden of cardiovascular diseases in the enlarged European Union”, European Heart Journal, 2006.

Mackaz J., Mensah G., “Atlas of Heart Disease and Stroke”, World Health Organisation Press, 2004. 
Migliavacca F., Petrini L., Montanari V., Quagliana I., Auricchio F., Dubini G., “A predictive study of the mechanical behavious of coronary stents by computer modelling", Medical Enigineering and Physics, vol. 27, 2005, p. 13-18.

Moens A.I., “Die Pulskurve”, Leiden, 1878.

Salehi M., "Biomechanical modelling of the arterial fluid-structure interaction in heamodynamocs", Fluid-structure interaction, (ASEN 5529), 2004.

Womersley J.R., "The mathematical analysis of the arterial circulation in a state of oscillatory motion", Wright Air Defence Centre, WADC TR56-614, 1957.

Young T., "Hydraulic investigations, subservient to an intended croonian lecture on the motion of blood Philosophical Transactions of the Royal Society (London)", vol. 96, 1808, p. $164-169$

Zhao S.Z., Xu X.Y., Collins M.W., "The numerical analysis of fluid-solid interactions for blood flow in arterial structures, Part 1: a review of models for arterial wall behaviour", Proc Instn Mech Engrs H, vol. 212, 1999, p. 229-240. 